\title{
Rectal application of argan oil improves healing of colorectal anastomosis in rats ${ }^{1}$
}

Aziz Mutlu Barlas', Serdar Kuru", Kemal Kismet'"', Turgut Cavusoglu'v, Yusuf Murat Bag ${ }^{\vee}$, Mehmet Senes ${ }^{\mathrm{V}}$, Neslihan Cihan VII, Pinar Celepli ${ }^{\mathrm{VIII}}$, Yilmaz Unal ${ }^{\mathrm{X}}$, Sema Hucumenoglu ${ }^{\mathrm{X}}$

'MD, General Surgeon, Ankara Education and Research Hospital, Department of General Surgery, Ankara, Turkey. Conception and design of the study.

"Associate Professor, Ankara Education and Research Hospital, Department of General Surgery, Ankara, Turkey. Design of the study and analysis of data.

I''Associate Professor, Ankara Education and Research Hospital, Department of General Surgery, Ankara, Turkey. Design of the study and manuscript writing.

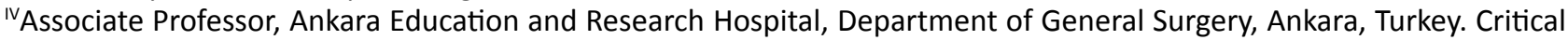
revision.

${ }^{\vee} \mathrm{MD}$, General Surgeon, Ankara Education and Research Hospital, Department of General Surgery, Ankara, Turkey. Design of the study.

${ }^{\mathrm{V}}$ Associate Professor, Ankara Education and Research Hospital, Department of Biochemistry, Ankara, Turkey. Performed the biochemical analyses.

VIIMD, Biochemist, Ankara Education and Research Hospital, Department of Biochemistry, Ankara, Turkey. Performed the biochemical analyses.

VIIIMD, Pathologist, Ankara Education and Research Hospital, Department of Pathology, Ankara, Turkey. Histopathological examinations.

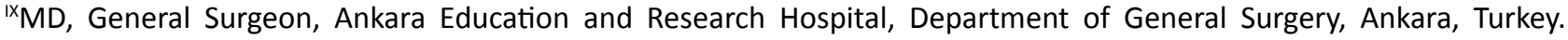
Conception of the study and interpretation of data.

xFull Professor, Ankara Education and Research Hospital, Department of Pathology, Ankara, Turkey. Histopathological examinations.

\section{Abstract}

Purpose: To investigate the possible effects of argan oil on the healing of colorectal anastomoses.

Methods: In Group 1 (sham), laparotomy was performed and the colon was mobilized. In the control (Group 2) and argan oil (Group 3) groups, colonic resection and anastomosis were applied. To the control and sham groups, $2 \mathrm{~mL}$ of $0.9 \% \mathrm{NaCl}$ was administred rectally, and in the argan oil group, $2 \mathrm{~mL} /$ day argan oil was applied rectally for 7 days.

Results: The mean bursting pressures of the argan oil and sham groups were significantly higher than the values in the control group. A significant difference was determined between the tissue hydroxyproline and prolidase levels of control group and other groups. Histopathologically, argan oil showed significant beneficial effects on colonic wound healing. In the argan oil and sham groups, the tissue malondialdehyde and fluorescent oxidation product levels were found to be lower and total sulfhydryl levels were higher than the control group. Conclusions: The rectally administered argan oil was observed to have significantly ameliorated wound healing parameters and exerted a significant antioxidant effect. This is the first study in the literature about the beneficial effects of argan oil on colorectal anastomoses.

Key words: Anastomosis, Surgical. Antioxidants. Argan. Rats. 


\section{Introduction}

Surgery is still the definitive treatment model for benign and malignant pathologies of the rectum and colon, such as diverticulosis, diverticulitis, inflammatory strictures, inflammatory bowel disease, volvulus, obstructive pathologies, fistulae and colorectal cancer. To improve oncological outcomes and quality of life of patients, colorectal surgical techniques have been gradually modified and some novel surgical techniques including laparoscopic and robotic surgery, extralevator abdominoperineal resection, and total mesorectal excision have been implemented in recent colorectal surgery. Anastomotic dehiscence, which leads to increased mortality and morbidity rates, prolonged hospitalization, higher costs, and higher cancer recurrence rates, is one of the most dramatic and frequent complications of colorectal operations. In spite of improvements in surgical techniques and increased experience, reported anastomotic dehiscence rates are still high ranging between $1 \%$ and $30 \%$. However, experienced and specialized colorectal surgeons have confirmed leakage rates of between 3\% and $6 \%$ as acceptable. It is generally accepted that emergent operations have a greater risk of the breakdown of anastomosis than elective procedures $^{1,2}$. Although several studies have been conducted over the years, not only for the development of new surgical techniques but also to evaluate the use of some locally applied or systemic products, there is still no widely accepted treatment method for the prevention of anastomotic leakage.

Argan oil has been used traditionally as a topical oil for the treatment of several diseases and conditions including acne, eczema, wrinkles, dry skin, joint pain, psoriasis, and skin inflammation. It is obtained from the fruits of the Argan tree (Argania spinose L.) which is a slow-growing tree, native to the southwest region of Morocco. Argan oil is also used as an ingredient in the cosmetic products such as moisturizers, shampoos, and other processed cosmetics. It is known to have antioxidant, anti-inflammatory, choleretic, hepatoprotective anti-acne, anti-sebum, antiobesity, antidiabetic, anti-aging, moisturizing, and cancer preventive properties ${ }^{3-7}$. Moreover, argan oil has been shown to be effective in healing experimentally created second-degree burns in rats ${ }^{8}$.

In the light of the beneficial effects of argan oil on wound healing, in addition to the antioxidant and anti-inflammatory effects, the aim of the current experimental study was to evaluate the possible effects of rectal administration of argan oil on colorectal anastomosis, and there is no study about this subject in the current literature.

\section{Methods}

All procedures in the existing experimental study were applied in compliance with the National Guidelines for the Use and Care of Laboratory Animals and were confirmed by the Animal Ethics Committee of of Ankara Education and Research Hospital (the date of approval: 24.11.2017, the protocol number: 0043).

The study was performed on 30 female Wistar-Albino rats, each weighing $250 \pm 25$ g. All rats were fed with commercial rat chow, and kept in wire cages and standard laboratory conditions. Food was withhold $12 \mathrm{hrs}$ before the operation and water was withdrawn $2 \mathrm{hrs}$ before anesthesia. During the study, no enteral or parenteral antibiotics were given. Rats that died throughout the experiment process were excluded from the study and no rat was added to the experimental groups.

Thirty rats that were included in the study were randomly divided in three groups. Anesthesia was maintained with an 
intramuscular injection of xylazine $5 \mathrm{mg} / \mathrm{per}$ $\mathrm{kg}$ and $50 \mathrm{mg} /$ per $\mathrm{kg}$ ketamine hydrochloride. All procedures were carried out under aseptic conditions. In the sham group (Group 1), only midline laparotomy and colonic externalization was performed and then the colon was replaced into abdomen. In the control and argan oil groups (Groups 2 and 3), colonic resection and end-to-end anastomosis were performed. A $2 \mathrm{~cm}$ section of the left colon, located 3 $\mathrm{cm}$ distance from the peritoneal reflection, was resected. Colonic anastomosis was then created using interrupted, single-layer, and inverting sutures with $5 / 0$ polydioxanone. Before closure of the abdominal incisions, $2 \mathrm{cc}$ of $0.9 \% \mathrm{NaCl}$ (in Groups 1 and 2) or argan oil (in Group 3) was applied to the suture lines. The abdomen was closed with $3 / 0$ silk continuous sutures. All procedures were carried out by the same surgeon. The argan oil used in the present study was supplied from Daleel Kozmetik San. Tic. A.S., Istanbul, Turkey (Mia Organical ${ }^{\circledR}, 100 \%$ Pure Argan Oil, Certificated by Etko-Cosmos Organic-5016-2015).

A $6 \mathrm{~F}$ feeding tube was inserted, approximately $5 \mathrm{~cm}$, into the rectum once a day for 7 days, beginning from the $1^{\text {st }}$ postoperative day. Using this feeding tube, $2 \mathrm{~mL}$ of $0.9 \%$ $\mathrm{NaCl}$ (in sham and control groups) or argan oil (in Group 3) was administered rectally. Before this administration process, the rats were anesthetized with $8 \mathrm{mg} /$ per kg ketamine hydrochloride intramuscularly. The rats were kept in the Trendelenburg position during the rectal administration of saline or argan oil solutions.

On postoperative $8^{\text {th }}$ day, all rats were sacrificed using high-dose anesthetic. Colonic segments, including sections $2 \mathrm{~cm}$ proximal and $2 \mathrm{~cm}$ distal of the anastomosis, were resected. First, the bursting pressures of the resected specimens were measured. Then the specimens were longitudinally divided into two pieces one of which was used for histopathological evaluation and the other for biochemical evaluations.

If the surrounding tissues were adhered tightly to the anastomosis site, these tissues were not separated and the specimen was removed en-bloc with the surrounding tissues. The distal end of the colonic segment was tied with $3 / 0$ silk suture. A feeding tube was inserted into the colonic segment from the proximal end. To avoid air leaks, this end was also tied using $3 / 0$ silk suture. The catheter in the segment was connected to a sphygmomanometer which contained a pump and a barometer. The segment was placed in a water-filled container. The pressure was gradually increased using the pump of the sphygmomanometer. When air bubbles appeared, that pressure was accepted as the bursting pressure and was recorded as millimeters of mercury $(\mathrm{mmHg})$.

The colonic specimens including the anastomosis site were stored in the Eppendorf tubes at $-80^{\circ} \mathrm{C}$ until assay. After weighing the tissues, $1 \mathrm{~mL}$ volume of $6 \mathrm{M}$ hydrochloric acid was added to $50 \mathrm{mg}$ of colonic tissues. The mixtures were hydrolyzed using an autoclave at $150^{\circ} \mathrm{C}$ for $1 \mathrm{~h}$. Ten microliters $(\mu \mathrm{L})$ of hydrolyzed mixture was transferred to other tubes and these were dried at room temperature. Chloramine-T solution of $0.2 \mathrm{~mL}$ was added to the tube, and after 10 mins, 1 $\mathrm{mL}$ of Ehrlich reagent was added and mixed. The mixtures were stored at $50^{\circ} \mathrm{C}$ for $90 \mathrm{~min}$. After this incubation period, the absorbance value of the mixtures were measured spectrophotometrically. ${ }^{9}$

After the colonic tissues were separated into small pieces, they were homogenized in distilled water to 10 times of the volume. The prepared homogenates were centrifuged at 800-g for $15 \mathrm{~min}$ and then the supernatant was collected for measurement of prolidase activity by the method described by Chinard. ${ }^{10}$

The tissues including the anastomotic site were fixed in $10 \%$ formalin. After fixing in 
paraffin, $4 \mu \mathrm{m}$-tissue sections were prepared. These preparations were stained with Masson's trichrome and hematoxylin\&eosin (H\&E) then examined under a light microscope by the pathologist who was blinded to the experimental groups. H\&E-stained tissue sections were evaluated by using the Ehrlich\&Hunt numerical scale. ${ }^{11}$ According to this scale, fibroblast content, neovascularization, inflammation, and collagen accumulation were scored as follows: 0: no evidence, 1: small amount and scattered, 2: small amount at all areas, 3: much, but scattered, and 4: dense at all areas. The presence or absence of a regular collagen fibrin pattern was evaluated on the preparations stained with Masson's trichrome.

The oxidative stress parameters were analysed in the Biochemistry Department of Ankara Education and Research Hospital. Tissue samples were kept at $-80^{\circ} \mathrm{C}$ till the analysis. The tissue malondialdehyde (MDA), total sulfhydryl (T-SH), and fluorescent oxidation product (FOP) levels were evaluated.

MDA levels were measured by a spectrofluorometric method, defined by Wasowicz et al. ${ }^{12}$, which is based on the calculation of the product of thiobarbutiric acid-MDA reaction at temperatures of 90$100^{\circ} \mathrm{C}$. Tissue homogenate of $50 \mathrm{~mL}$ and $50 \mathrm{~mL}$ of standard MDA assay solutions were added to the tubes containing $1 \mathrm{~mL}$ of distilled water. TBA prepared in $1 \mathrm{~mL} 29 \mathrm{mmol} / \mathrm{L}$ acetic acid was added to the tubes which were then left for $1 \mathrm{~h}$ incubation in a $95-100^{\circ} \mathrm{C}$ water bath. After this incubation period, $5 \mathrm{~mol} / \mathrm{L} \mathrm{HCl}(25 \mu \mathrm{L})$ and then $\mathrm{n}$-butanol $(3.5 \mathrm{~mL}$ ) were added to the samples and the process of extraction was utilized by mixing the tubes for 5 min with a vortex. After centrifugation of the tubes for $10 \mathrm{~min}$ at 1500 $\mathrm{g}$, the fluorescence of the extract was read on a Hitachi F-2500 spectrofluorometer at wavelengths of $525 \mathrm{~nm}$ and $547 \mathrm{~nm}$.

Total SH levels were determined using spectrophotometrical method defined by
Sedlak and Lindsay. ${ }^{13}$ The supernatant part of the tissue homogenate $(250 \mu \mathrm{L})$ was mixed with Tris buffer $(750 \mu \mathrm{L}-0.2 \mathrm{M})$, and $50 \mu \mathrm{L}$ volume of 5,5"-dithiobis (2-nitrobenzoic acid) (0.01 M DTNB). Adding $3950 \mu \mathrm{l}$ of methanol, the volume of the mixture was achieved to $5 \mathrm{~mL}$. A sample blank without DTNB and a reagent blank without sample were also prepared in a similar way. After a 15-min incubation period, the mixtures were centrifuged at $3000 \mathrm{~g}$ for 15 minutes at room temperature. The absorbance of clear supernatant was measured at $412 \mathrm{~nm}$ in a spectrophotometer (Schimadzu CL-770, Kyoto, Japan).

For FOP measurements, homogenated tissues including the anastomotic site were extracted using ethanol-ether. These homogenates were then calculated through a spectrofluorometer at wavelengths for excitation/emission. ${ }^{14}$

Data analysis was performed using the Statistical Package for Social Sciences version 15.0 (SPSS Inc, Chicago, IL). The data were given as mean \pm standard deviation. Differences between the data of the groups were evaluated using Kruskal-Wallis variance analysis or OneWay variance analysis (ANOVA). If the varience analysis revealed a significant difference, the Mann-Whitney $U$ multiple comparison test or Tukey honestly significant difference was used to determine the difference between the groups. A value of $p<0.05$ was accepted as statistically significant.

\section{- Results}

One rat from the sham group and 2 rats from the argan oil group died in the early postoperative period possibly due to anesthesia complications. Thus, the study was completed with the results of the remaining 27 rats. No rats were found to have abscess or free perforation.

The mean colonic bursting pressures 
are given in Table 1 . The mean bursting pressures of the argan oil $(p=0.017)$ and sham $(p=0.009)$ groups were significantly higher than the values in the control group. The hightest bursting pressures were determined in the sham group (Group 1).

Table 1 - The mean colonic bursting pressures of the groups.

\begin{tabular}{ll}
\hline Groups & $\begin{array}{l}\text { Colonic bursting pressures } \\
(\mathrm{mmHg})\end{array}$ \\
\hline Sham $(\mathrm{n}=9)$ & $178.75 \pm 16.42$ \\
Control $(\mathrm{n}=10)$ & $123.50 \pm 66.50^{\mathrm{a}, \mathrm{b}}$ \\
Argan oil $(\mathrm{n}=8)$ & $174,44 \pm 22.42$ \\
\hline
\end{tabular}

${ }^{\mathrm{a}}$ significantly different, $\mathrm{p}<0.05$, sham vs. control, ${ }^{\mathrm{b}}$ significantly different, $p<0.05$, control vs. argan oil.
The hydroxyproline levels, a marker of collagen content, are presented in Table 2. The highest levels were determined in the sham group. There was no significant difference between the sham and argan oil groups ( $p>0.05)$. A significant difference was determined between the control group (Group $1)$ and the argan oil ( $p=0.002)$ and sham $(p=0.001)$ groups.

The mean tissue prolidase levels are shown in Table 2. The lowest mean prolidase level was found in the sham group. No significant difference was found between the sham and argan oil groups $(p>0.05)$. A significant difference was found between the control group and the other groups $(p=0.011$ argan oil, $p=0.01$ sham).

Table $\mathbf{2}$ - The mean hydroxyproline and prolidase levels of the groups.

\begin{tabular}{lll}
\hline Groups & Hydroxyproline (uM/g protein) & Prolidase (U/g protein) \\
\hline Sham $(n=9)$ & $91.26 \pm 13.52$ & $181.96 \pm 65.57$ \\
Control $(n=10)$ & $62.02 \pm 9.32^{\mathrm{a}, \mathrm{b}}$ & $310.42 \pm 86.03^{\mathrm{a}, \mathrm{b}}$ \\
Argan oil $(n=8)$ & $89.49 \pm 17.01$ & $199.35 \pm 53.72$ \\
\hline
\end{tabular}

${ }^{a}$ significantly different, $p<0.05$, sham $v s$. control, ${ }^{b}$ significantly different, $p<0.05$, control vs. argan oil.

The mean histopathological scores are given in Table 3 . The scores of sham group was significantly different from the scores of control group for all parameters $(p<0.05)$ and also different from argan oil group $(p<0.05)$ for all parameters except for inflammation scores. When the argan oil and control groups were compared, it was detected that argan oil showed significant beneficial effects on colonic wound healing, demonstrated by inflammation, fibroblast and collagen scores $(p=0.001$ for these parameters). In the sham group, the colonic structure was normal histopathologically (Figure 1). In the argan oil group, inflammatory cell infiltration was significantly less, fibroblasts and collagen accumulation were more intense than the control group (Figures 2 and 3, H\&E and Masson's trichrome stainings).

Table 3 - The mean histopathological scores.

\begin{tabular}{lllll}
\hline Groups & Inflammation & Fibroblast & Neoascularisation & Collagen \\
\hline Sham $(n=9)$ & $0.71 \pm 0.35$ & $0.00 \pm 0.00$ & $0.14 \pm 0.04$ & $0.00 \pm 0.00$ \\
Control $(n=10)$ & $2.75 \pm 0.46^{\mathrm{a}}$ & $0.88 \pm 0.34^{\mathrm{a}}$ & $1.50 \pm 0.72^{\mathrm{a}}$ & $0.88 \pm 0.34^{\mathrm{a}}$ \\
Argan oil $(n=8)$ & $1.12 \pm 0.64^{\mathrm{c}}$ & $2.50 \pm 0.53^{\mathrm{b}, \mathrm{c}}$ & $2.00 \pm 0.75^{\mathrm{b}}$ & $2.50 \pm 0.53^{\mathrm{b}, \mathrm{c}}$ \\
\hline
\end{tabular}

${ }^{a}$ significantly different, $p<0.05$, sham vs. control, ${ }^{b}$ significantly different, $p<0.05$, sham vs. argan oil, ' significantly different, $p<0.05$, control vs. argan oil. 


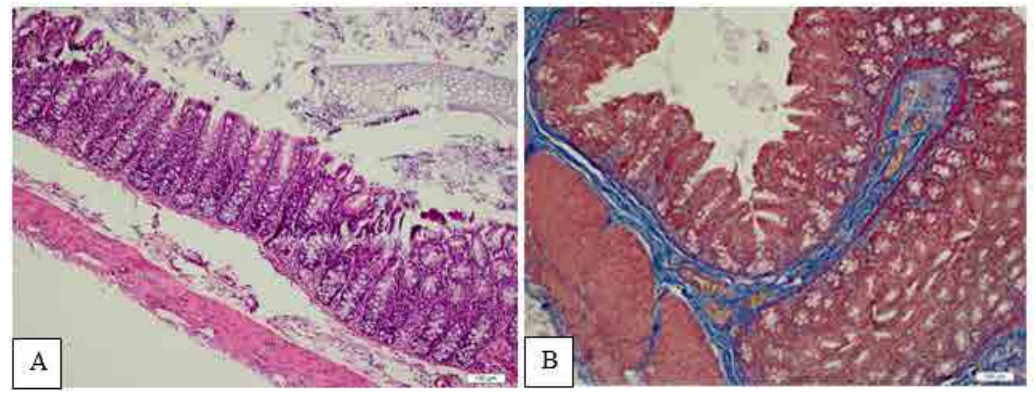

Figure 1 - A. and B. Inflammatory cells, fibroblastic activity, neovascularization and collagen accumulation are not observed in the sham group (H\&E, and Masson's Trichrome).
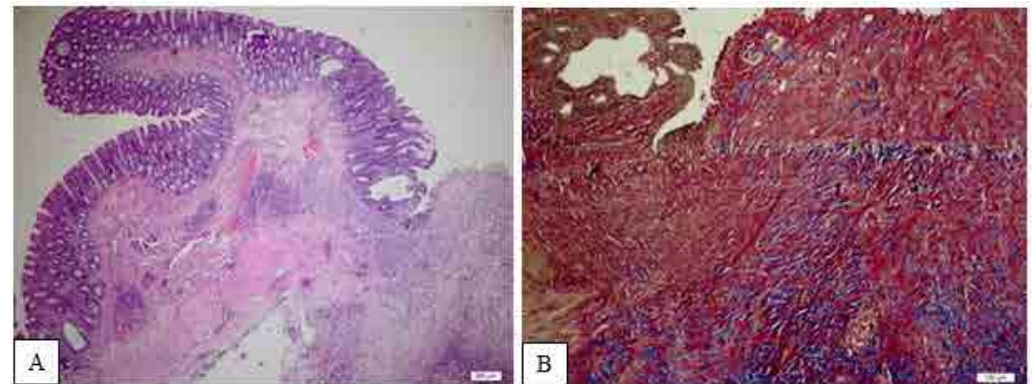

Figure $\mathbf{2}$ - A. In the control group, inflammatory cell infiltration is more and angiogenesis is less. (H\& E). B. Masson's Trichrome staining shows less collagen accumulation.
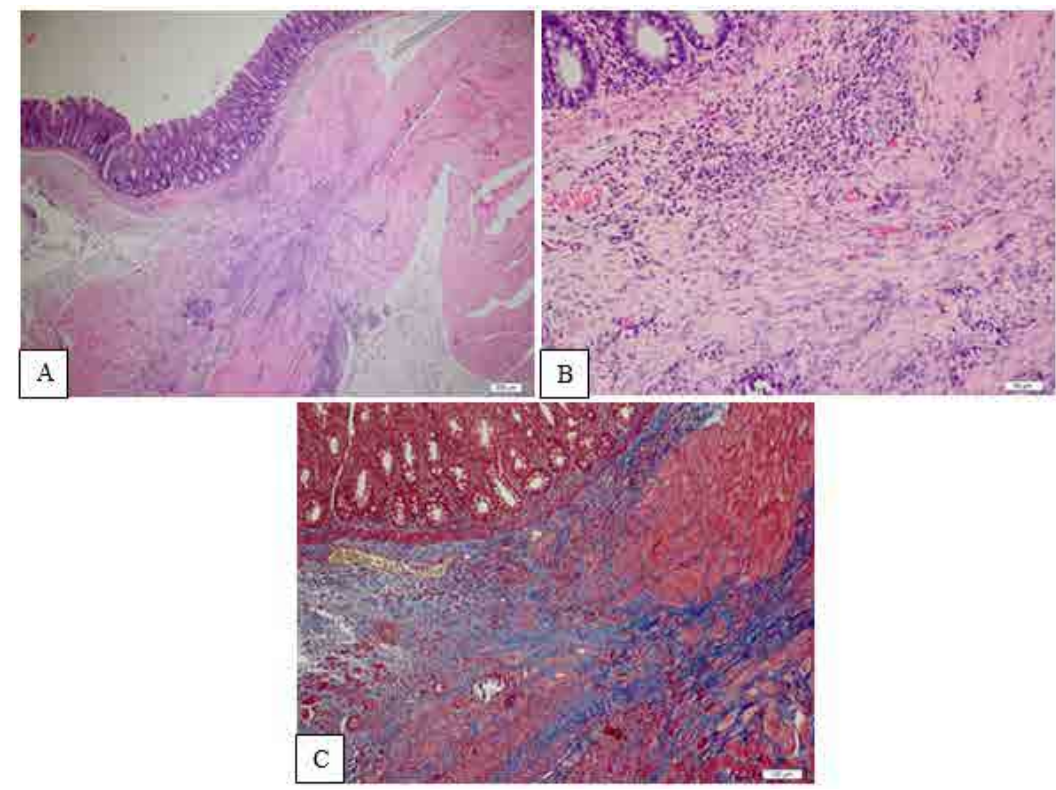

Figure 3 - A. and B. In the argan oil group, inflammatory cells are less and neovascularization is observed $(\mathrm{H}$ \& E), C. Intense collagen accumulation in the argan oil group (Masson's Trichrome).

The mean values of the oxidative stress parameters are presented in Table 4 . In the argan oil and sham groups, the MDA and FOP levels were found to be lower and T-SH levels were higher when compared with the control group ( $p<0.05$ for all parameters). No significant difference was found between the sham and argan oil groups ( $p>0.05$ for all parameters). 
Table 4 - Oxidative stress parameters of the groups.

\begin{tabular}{llll}
\hline Groups & MDA (nmol/g protein) & T-SH (umol/g protein) & FOP (FOP/g protein) \\
\hline Sham $(n=9)$ & $24.99 \pm 12.87$ & $31.74 \pm 6.62$ & $92.37 \pm 32.36$ \\
Control $(n=10)$ & $81.75 \pm 38.56^{\mathrm{a}, \mathrm{b}}$ & $18.04 \pm 2.23^{\mathrm{a}, \mathrm{b}}$ & $144.86 \pm 37.64^{\mathrm{a}, \mathrm{b}}$ \\
Argan oil $(\mathrm{n}=8)$ & $29.48 \pm 8.65$ & $26.07 \pm 4.21$ & $94.85 \pm 18.64$ \\
\hline
\end{tabular}

MDA: Malondialdehyde, T-SH: Total sulfhydryl, FOP: Fluorescent oxidation product.

${ }^{\mathrm{a}}$ significantly different, $\mathrm{p}<0.05$, sham vs. control, ${ }^{\mathrm{b}}$ significantly different, $\mathrm{p}<0.05$, control vs. argan oil.

\section{- Discussion}

Anastomotic leakage following resection and anastomosis, which causes increased mortality and morbidity rates and prolonged length of stay in hospital, is still an important and serious complication of colorectal surgery with unacceptably high rates between $6 \%$ and $30 \%$. Many risk factors are known to be associated with anastomotic leakage including patient factors such as obesity, malnutrition, male gender, cardiovascular disease, steroids, ASA (American Society of Anesthesiologists) score, tobacco and alcohol use, diverticulitis, leukocytosis, and operative factors such as low rectal anastomosis, bowel obstruction, perioperative blood transfusion, operative time more than 2 hours, suboptimal blood supply to anastomosis, and intraoperative septic conditions. Despite new tools used for bowel anastomosis, developments in surgical technology, improvements in the treatment of surgical infections, better information about the risk factors of anastomotic leaks, usage of different solutions for preventing anastomotic leakage, and improved perioperative care, anastomotic leakage remains a major problem and the optimal treatment for preventing this serious complication has not been clearly identified yet ${ }^{2,15-17}$.

Argan oil has been utilized as a food and food ingredient, and for centuries has been used topically for the treatment of several skin diseases and for cosmetic purposes. Its chronic and acute toxicity, especially when administered orally at common doses, is minimal, almost nil. The pharmacological properties of argan oil are generally depends on the properties of its constituents that have been isolated and then evaluated pharmacologically ${ }^{18}$. Virgin argan oil is composed of $99 \%$ acylglycerides. The remaining $1 \%$, unsaponifiable matter, is composed of tocopherols, carotenes, sterols, triterpene alcohols, and xanthophylls. Fatty acids which compose acylglycerides are mainly oleic (43-49\%) and linoleic acid (29-36\%) Palmitic acid and stearic acid are the other fatty acids in the argan oil in small amounts. Other organic derivatives in argan oil are phenols, the concentration of which are very low, including vanillic acid, ferulic acid, syringic acid, catechol, catechin tyrosolresorcinol, and epicatechin. There are also many other minor organics in argan oil such as squalene, triterpene, carotenes, alcohols, sterols, etc. Most of the pharmacological properties of argan oil are thought to be due to its high unsaturated fatty acid content. The most important properties of argan oil comprise the numerous therapeutic effects of oleic acid, which is a monounsaturated fatty acid. As the deficiency of linoleic acid can cause inadequate wound healing, the traditional usage of argan oil for skin inflammation may be attributed to its high linoleic acid content ${ }^{18}$.

Many specific health benefits of argan oil are contributed by its unsaponifiable matter content and high tocopherol content which is 
high in argan oil $(620 \mathrm{mg} / \mathrm{kg})$ when compared with olive oil $(320 \mathrm{mg} / \mathrm{kg})$. Tocopherols have strong free radical scavenging and antioxidant properties. The most effective free radical scavenger of all tocopherols, gammatocopherol, constitutes $69 \%$ of the total tocopherol content of argan oil. As tocopherols and phenolic compounds can act synergistically, and they have significant antioxidant and freeradical scavenging properties, these molecules are thought to be responsible for most of the therapeutic effects of argan oil. Phytosterols and phenolics also possess indirect or direct hypocholesterolemic and hypolipidemic activity ${ }^{3,18}$.

Apart from the usage of argan oil for cosmetic purposes, there have been several studies evaluating the beneficial effects of argan oil on different pathological conditions, not only through systemic administration but also with local application. Necib et al. ${ }^{4}$ investigated the effects of an argan oil and sodium selenite combination on mercuric chloride toxicity in the rat liver and found that this combination restored the increased liver functions and high lipid peroxidation levels. This supplementation also decreased the morphological damage in the rat liver caused by mercuric chloride and strongly protected the liver from this toxicity. Sekeroglu et al. ${ }^{5}$ investigated the protective effects of another well-known toxicant, acrylamide, the source of which is smoking and the foods that are processed at high-temperatures. The authors showed that argan oil reduced genetic damage, emperipolesis, and oxidative stress in acrylamide-induced toxicity and suggested that argan oil could be used against diseases related to oxidative stress. In another study it was demonstrated that argan oil modulated the antioxidant systems and mitochondrial function in the rat brain ${ }^{6}$.

In addition to these experimental studies, argan oil has been used in the human studies. Eljaoudi et al. ${ }^{7}$ gave hemodialysis patients $30 \mathrm{~mL} /$ day virgin argan oil for a 4-week period and evaluated the effect of argan oil on antioxidant status and lipid profile. Virgin argan oil consumption was determined to have decreased total cholesterol, triglyceride, and low-density lipoprotein levels and increased high-density lipoprotein levels. Malondialdehyde levels increased after the hemodialysis sessions, but this increase was lower in the argan oil group compared with the control group. Plasma vitamin E levels increased significantly from baseline in the argan oil group. The authors concluded that virgin argan oil improved oxidative stress status and lipid profile in end-stage renal disease patients.

Moreover, Avsar et al. ${ }^{8}$ investigated the effects of argan oil on wound healing in an experimental second-degree burn model. Treatment of the burn injuries was started 24 hours after the injury, and a $1 \mathrm{~mL}$ dose of argan oil was applied to the wounds by using a syringe. The wound healing rates were evaluated on post-injury days 1,7 , and 14. Tissue samples were determined for histological and molecular changes in fibroblast activity and TGF- $\beta$ expression. The contraction of burned tissue was also calculated. The mRNA levels of TGF- $\beta 1$ were significantly increased in the group administered argan oil twice a day when compared with the control group. The contraction rates were increased in both the argan oil groups (once and twice a day groups) at all 3 timepoints. When compared with the silver sulfadiazine group, the twice-a-day argan oil group showed greater rates of contraction. Histopathological examinations showed better healing and contraction evidence in the argan oil groups and the silver sulfadiazine group compared to the control group. The authors suggested that argan oil was effective on 
second-degree burns in rats.

In the light of the above-mentioned studies which confirmed the antioxidant, antiinflammatory and wound-healer effects of argan oil, the current study was planned to determine the effects of argan oil administered rectally on the colonic anastomotic healing in a rat colonic resection-anastomosis model.

In animal models, bursting pressure is the most commonly used marker of colorectal anastomotic healing. Although there are several methods to evaluate bursting pressure, the most accepted test for this purpose is inflation of the anastomosed bowel segment with a pump and accepting the pressure at which the air leakage is seen at the anastomotic site, as bursting pressure. However, there are some ongoing arguments on the evaluation of bursting pressure. First, some authors have concluded that disrupting tissue specimens makes the histopathological evaluation difficult. Second, it has also been stated that bursting pressure is not an appropriate indicator since it can not be used for previously ruptured and leaky anastomoses. Although these arguments are ongoing, bursting strength measurement is still widely used for the evaluation of anastomotic strength in animal studies. Quantitative comparison of bursting pressures between different studies is not valid because of the usage of various protocols and instruments. Therefore, bursting pressure values should be compared only between experimental groups of the same study ${ }^{19}$. In the current study, the bursting pressure values were used for the comparison of anastomotic strength between the groups. The mean bursting pressure level of the argan oil group was significantly higher than the levels in the control group $(p=0.006)$. No statistically significant difference was found between the bursting pressures of the sham and argan oil groups. These results showed that the application of argan oil increased the anastomotic strength and reduced the leakage risk.

Histopathological examination is another procedure for evaluating the colorectal anastomotic healing in experimental studies. The grading scales used for this evaluation are generally based on cutaneous wound healing including fibroblast activity, inflammatory cell infiltration, collagen deposition and neoangiogenesis or vascularity ${ }^{19}$. In the current study, argan oil exhibited beneficial effects on the histopathological parameters. The collagen accumulation and fibroblast proliferation were more intense, and inflammation was significantly less in the argan oil group when compared with histopathological findings of the control group.

Collagen is the most critical molecule maintaining anastomotic wall strength, therefore, measuring collagen content at the anastomotic site has been used as another important marker of anastomotic healing. Hydroxyproline, together with proline, plays an important role in the stability of collagen. The evaluation of collagen content is generally performed by calculating levels of hydroxyproline, the amino acid which exists in limited proteins other than collagen. Although hydroxyproline levels do not provide information about collagen subtypes and the maturity of collagen, this marker is widely used to provide an opinion about the collagen content of tissues in association with anastomotic healing in experimental colorectal anastomosis models ${ }^{19,20}$. In the current study, the levels of hydroxyproline, which is a marker of collagen content of the anastomotic site, were significantly higher in the argan oil group than in the control group $(p=0.002)$ indicating that anastomotic healing was better in the argan oil group.

$$
\text { Prolidase, the cytosolic }
$$

imidodipeptidase, is an enzyme which 
splits iminodipeptides containing carboxyterminal hydroxyproline or proline. It has an important role in matrix remodelling, collagen metabolism, and cell growth. Imidodipeptides are derived from the degradation of procollagen, collagen, other proteins containing collagen, and dietary proteins. Prolidase plays a significant role in splitting proline from imidodipeptides for synthesis of collagen and other prolinecontaining proteins. Prolidase deficiency is an autosomal recessive disorder characterized by severe imidodipeptiduria and increased plasma levels of proline-containing dipeptides with various symptoms, such as mental retardation, recurrent infections, skin lesions, and collagen tissue abnormalities ${ }^{21,22}$. In the current study, the prolidase levels were statistically significantly higher in the control group than in the argan oil and sham groups ( $p<0.05$ for both groups). Although the prolidase levels in the argan oil group were higher than the levels in the sham group, there was no significant difference between these groups ( $p>0.05$ ). Increased prolidase activity in the control group can be considered to have resulted from the above-mentioned process of proline recycling to increase collagen resynthesis. In contrast, the low levels of prolidase activity in the argan oil groups might indicate the collagen stability and decreased rates of collagen re-synthesis. In addition, no significant difference was detected between the sham and argan oil groups according to prolidase activities ( $p>0.05$ ).

In order to detect the possible mechanism that is responsible for the beneficial effects of argan oil on wound healing, the oxidative stress parameters were evaluated. In the argan oil group, MDA and FOP levels were found to be lower and T-SH levels were higher when compared with the control group $(p<0.05$ for all parameters). No significant difference was found between the sham and argan oil groups ( $p>0.05$ for all parameters). These results showed that argan oil had significant antioxidant properties and this might be one of the possible mechanisms that is responsible for the beneficial effects.

In the current study, argan oil was applied rectally and daily for 7 days. This route was preferred because rectal administration provides a longer period of contact with the anastomotic line than the local application on anastomotic line during surgery. It was applied for 7 days which is known to be optimal period for healing of colorectal anastomoses. Considering the width of rat colon, it was predicted that the application volume which would not disrupt the anastomosis was about $2 \mathrm{cc}$. The procedure used in this study was also used in a previous study of our research group ${ }^{20}$. However, further studies, which evaluate the different doses and concentrations of argan oil ascertaining the optimal effective dose with minimal adverse events, are needed.

\section{Conclusions}

The rectal administration of argan oil ameliorated bursting pressures, histopathological findings, prolidase activity and hydroxyproline levels in an experimental colorectal anastomosis model. The positive effects of argan oil on anastomotic healing could be considered to be based on its antioxidant and anti-inflammatory properties.

\section{References}

1. Stergios K, Kontzoglou K, Pergialiotis V, Korou LM, Frountzas M, Lalude O, Nikiteas N, Perrea DN. The potential effect of biological sealants on colorectal anastomosis healing in experimental research involving severe diabetes. Ann R Coll Surg Engl. 2017;99:189- 
92. doi: 10.1308/rcsann.2016.0357.

2. Kingham TP, Pachter HL. Colonic anastomotic leak: risk factors, diagnosis, and treatment. J Am Coll Surg. 2009;208:269-78. doi: 10.1016/j.jamcollsurg. 2008. 10. 015.

3. Guillaume D, Charrouf Z. Argan oil. Monograph. Altern Med Rev. 2011;16:2759. PMID: 21951028.

4. Necıb Y, Bahi A, Zerizer S. Amelioration of mercuric chloride toxicity on rat liver with argan oil and sodium selenite supplements. Int J Pharm Bio Sci. 2013,4:839-49.

5. Şekeroğlu ZA, Aydın B, Şekeroğlu V. Argan oil reduces oxidative stress, genetic damage and emperipolesis in rats treated with acrylamide. Biomed Pharmacother. 2017;94:873-9. doi: 10.1016/j. biopha.2017.08.034.

6. Aydın B. Effects of argan oil on the mitochondrial function, antioxidant system and the activity of NADPH-generating enzymes in acrylamide treated rat brain. Biomed Pharmacother. 2017;87:476-81. doi: 10.1016/j.biopha.2016.12.124.

7. Eljaoudi R, Elkabbaj D, Bahadi A, Ibrahimi A, Benyahia M, Errasfa M. Consumption of argan oil improves anti-oxidant and lipid status in hemodialysis patients. Phytother Res. 2015;29:1595-9. doi: 10.1002/ ptr.5405.

8. Avsar U, Halici Z, Akpinar E, Yayla M, Avsar U, Harun U, Harun U, Tarik AH, Bayraktutan Z. . The effects of argan oil in second-degree burn wound healing in rats. Ostomy Wound Manage. 2016;62:26-34. PMID: 26978857.

9. Jamall IS, Finelli VN, Que Hee SS. A simple method to determine nanogram levels of 4-hydroxyproline in biological tissues. Anal Biochem. 1981;112:70-5. PMID: 7258630 .

10.Chinard FP. Photometric astimation of proline and ornithine. J Biol Chem. 1952;199:91-5. PMID: 12999819.

11.Phillips JD, Kim JS, Fonkalsrud EW, Zeng $H$, Dindar $\mathrm{H}$. Effects of chronic corticosteroids and vitamin $A$ on the healing of intestinal anastomoses. Am J Surg. 1992;163:71-7. PMID: 1733376.

12.Wasowicz W, Neve J, Peretz A. Optimized steps in fluorometric determination of thiobarbituric acid-reactive substances in serum: importance of extraction $\mathrm{pH}$ and influence of sample preservation and storage. Clin Chem. 1993;39:2522-6. PMID: 8252725.

13.Sedlak J, Lindsay RH. Estimation of total protein bound and non-protein sulphydryl groups in tissue with Ellman's reagen. Anal Biochem. 1968;25:192-205. PMID: 4973948.

14. Wu T, Willett WC, Rifai N, Rimm EB. Plazma fluorescent oxidation products as potential markers of oxidative stress for epidemiologic studies. Am J Epidemiol. 2007;165:552-60. doi: 10.1093/aje/kwm119.

15. Thomas MS, Margolin DA. Management of colorectal anastomotic leak. Clin Colon Rectal Surg. 2016;29:138-44. doi: 10.1055/ s-0036-1580630.

16.Nasir Khan MU, Abir F, Longo W, Kozol R. Anastomotic disruption after large bowel resection. World J Gastroenterol. 2006;12:2497-504. PMID: 16688793.

17.Shogan BD, Carlisle EM, Alverdy JC, Umanskiy $K$. Do we really know why colorectal anastomoses leak? J Gastrointest Surg. 2013;17:1698-707. doi: 10.1007/ s11605-013-2227-0.

18. Monfalouti HE, Guillaume D, Denhez C, Charrouf Z. Therapeutic potential of argan oil: a review. Pharm Pharmacol. 2010;62:1669-75. doi: 10.1111/j.20427158.2010. 01190.x.

19.Bosmans JW, Jongen AC, Bouvy ND, Derikx JP. Colorectal anastomotic healing: why the biological processes that lead to anastomotic leakage should be revealed prior to conducting intervention studies. BMC Gastroenterol. 2015;15:180. doi: 10.1186/s12876-015-0410-3.

20.Kuru S, Kismet K, Bag YM, Barlas AM, Senes M, Durak M, Yumusak N, Urhan MK, Cavusoglu T, Pekcici R. Does the application of Ankaferd Blood Stopper rectally have positive effects on the healing of colorectal anastomosis and prevention of anastomotic leakage? An experimental study. Biomed Pharmacother. 2017;96:968-73. doi: 10.1016/j.biopha.2017.11.140.

21. Namiduru ES. Prolidase. Bratisl Med. J 2016;117:480-5. PMID: 27546702. 
22.Surazynski A, Miltyk W, Palka J, Phang JM. Prolidase-dependent regulation of collagen biosynthesis. Amino Acids. 2008;35:731-8. doi: 10.1007/s00726-008-0051-8.

\section{- Acknowledgement}

This text has been proof-read and edited by Caroline Jane Walker, a university graduate English native-speaker, with over 10 years of experience in the field of translating, proof-reading and editing for medical journals.

\section{Correspondence:}

Kemal Kismet

SB Ankara Egitim ve Arastirma Hastanesi

Genel Cerrahi Klinigi, 06340, Ulucanlar

Ankara Turkey

Phone: +90.312.595 3885

kemalkismet@yahoo.com

Received: Mar 22, 2018

Review: May 24, 2018

Accepted: June 20, 2018
Conflict of interest: none

Financial source: none
${ }^{1}$ Research performed at Ankara Education and Research Hospital, Department of General Surgery, Ankara, Turkey. 